Rev. salud pública. 13 (2): 239-252, 2011

\title{
Representações de mulheres em idade fértil e profissionais de saúde sobre utilização de cuidados de saúde reprodutiva
}

\author{
Representations of fertile aged women and health professionals \\ about reproductive healthcare use
}

Isabel Craveiro y Paulo Ferrinho

Instituto de Higiene e Medicina Tropical. Universidade Nova de Lisboa. Lisboa, Portugal. isabelc@ihmt.unl.pt,pfeninho@ihmtunl.pt

Receivido 15 Julho 2010/ Enviado para Modificação 30 Octubre 2010/ Aprovado 12 Decembro 2010

\section{RESUMO}

Objectivos Conhecer as representações de mulheres pobres e não pobres relativamente à fecundidade verificando de que forma influenciam a utilização de cuidados de saúde reprodutiva (saúde materna e planeamento familiar). E as representações dos profissionais de saúde sobre comportamentos de fecundidade e forma de utilização de cuidados de saúde reprodutiva dessas mulheres.

Métodos Foi realizado um estudo qualitativo, tendo sido efectuadas oito entrevistas e dois grupos focais a mulheres provenientes de diferentes contextos socioeconómicos, num total de dezoito indivíduos. E dois grupos focais a profissionais de saúde (enfermeiros e médicos), num total de quinze participantes. Foi efectuada análise de conteúdo, tendo-se procedido a uma análise categorial temática.

Resultados Encontramos semelhanças entre as mulheres provenientes de diferentes gradientes sociais, mas também diferenças acerca do papel do parceiro masculino no planeamento familiar e das gravidezes. Os profissionais de saúde atribuem ao "pobre" uma característica-tipo: o imediatismo, que condiciona a actuação dos indivíduos "pobres" nas práticas de planeamento familiar e nas formas de utilização da saúde materna.

Conclusão A análise reflecte a existência de representações nem sempre coincidentes entre mulheres e profissionais de saúde, no que diz respeito à fecundidade e às necessidades e formas de utilização dos cuidados de saúde reprodutiva. É importante os decisores terem estes factos em atenção para adequar as políticas de saúde às expectativas e percepções de necessidade por parte das populações vulneráveis, procurando uma utilização adequada de cuidados de saúde reprodutiva e promover a equidade em saúde.

${ }_{1}^{1}$ Adaptado de la ponencia presentada por los autores en la $5^{\text {th }}$ International Conference de la International Society for Equity on Health, realizada en Creta, Grecia, Junio 9-11 de 2009. 
Palavras-chave: Medicina reprodutiva, percepção social, equidade em saúde, gênero e saúde (fuente: DeCS, BIREME).

\section{ABSTRACT}

Objective Ascertaining poor and not poor women'srepresentations about fecundity and verifying how this affectedreproductive health care use (maternal health care and family planning), as well ashealthcare professionals'representationsabout "poor" womenfecundity patternsand reproductive healthcare use.

Methods A qualitative study was carried out through interviews and focus groups. Specifically, eight interviews were held and two focus groups were run, giving a total of eighteen women from various socioeconomic backgrounds (living in poverty and not living in poverty). Both focus groups were run with healthcare professionals (nurses and doctors), giving a total of fifteen participants. Data was analysed by using content analysis identifying significant themes.

Results Similarities were found between women from different social backgrounds, although some differences were also found, particularly about male partners'rolein family planning and planning pregnancies. Health professionals' attributedstandard featuresto "poor" individuals,i.e. immediacy, conditioning family planning practice and maternal health care use patterns.

Conclusions The women and health professionals'representationsregarding fecundity, needs and ways of using reproductive health care (maternal health care and family planning) did not always coincide. Decision-makers'importance in realizing this fact must be emphasizedso that healthcare policies can be adaptedto vulnerable populations'texpectations and perceptions of need, thereby leading to the suitable use of reproductive healthcare and ultimately promoting equity in health.

Key Words: Reproductive medicine, social perception, inequality, health, women (source: MeSH, NLM).

RESUMEN

Representaciones de mujeres en edad reproductiva y de profesionales de la salud sobre la utilización de la atención en salud reproductiva

Objetivo Conocer las representaciones de las mujeres pobres y no pobres sobre la fecundidad y examinar cómo estas afectan la atención en salud reproductiva (salud materna y planificación familiar). Y las representaciones de los profesionales de la salud sobre las conductas de fecundidad y la atención en salud reproductiva de las mujeres.

Métodos Estudio cualitativo, por medio de ocho entrevistas y dos grupos focales de mujeres provenientes de diferentes contextos socioeconómicos, con un total de 18 personas. Se realizaron 2 grupos focales con profesionales de la salud (enfermeras y médicos), con un total de 15 participantes. Se utilizó el análisis de contenido por medio de categorías temáticas.

Resultado Se encontraron similitudes entre mujeres de diferentes gradientes sociales, aunque hubo diferencias particularmente en el papel de los compañeros hombres en la planificación familiar y de los embarazos. Los profesionales de la salud atribuyen a la pobreza una característica-tipo: el inmediatismo que condiciona la actuación de los individuos "pobres" en la práctica de la planificación familiar y en las formas de utilización de la atención materna.

Conclusión Las representaciones de las mujeres y de los profesionales de la salud sobre la fecundidad, las necesidades y formas de utilización de la atención en salud reporductiva, no siempre coinciden. Los tomadores de decisiones deben considerar estos hechos con el fin de adaptar las políticas de salud a las expectativas y percepciones de necesidades por parte de de las 
poblaciones vulnerables, procurando la utilización adecuada de la atención en salud reproductiva y promover la equidad en salud.

Palabras Clave: Medicina reproductiva, percepción social, equidad en salud, genero y salud (fuente: DeCS, BIREME).

$\mathrm{O}$ s estudos sobre atitudes e práticas são cada vez mais usados no âmbito la saúde pública, mas não especificamente relacionados com as representações e motivações para a utilização de cuidados de saúde materna e planeamento familiar, entre mulheres em idade fértil, vivendo em áreas urbanas e em contextos de pobreza e não pobreza.

Em Portugal, apesar dos enormes avanços nos indicadores de saúde (e.g., a diminuição nas taxas de mortalidade infantil e materna e o aumento na esperança de vida), continuam a verificar-se desigualdades na saúde e na acessibilidade aos serviços de saúde (1). Uma das estratégias gerais orientadoras do Plano Nacional de Saúde (PNS) é "a prioridade aos mais pobres", porém num estudo patrocinado pela Direcção Geral de Saúde (2) é reconhecido que a "... Possível relação entre as desigualdades sociais e a estratificação social com o género, enquanto determinante da saúde, é estabelecido de forma ténue". Uma das excepções mencionadas é o PNS, ao identificar a necessidade de haver um cruzamento conceptual e estratégico entre a estratificação social e a saúde das mulheres.

As representações sociais situam-se, do ponto de vista analítico, no domínio das teorias do senso comum ("Lay Theories") (3). Denise Jodelet apresenta uma definição de representações sociais, que se mantém das mais citadas: "É uma forma de conhecimento, socialmente elaborada e partilhada, tendo um lado prático, contribuindo para a construção de uma realidade comum a um grupo social" (4). Esta foi a perspectiva teórica por nós utilizada no contexto deste estudo.

O estudo das representações das mulheres e dos profissionais de saúde é importante para ter elementos de compreensão da mudança social e poder alimentar os debates sobre as políticas promotoras de equidade. Contribuindo para uma organização dos serviços de saúde mais adequada à realidade de quem os procura e consciente das representações de quem os presta.

Referências conceptuais

É hoje reconhecida a importância de abordar as questões relativas à pobreza, determinantes sociais e equidade, reconhecendo-se que as desigualdades socioeconómicas são um importante problema de saúde pública (5-8). 
Em termos empíricos seguimos a definição e operacionalização do conceito de pobreza defendidas por Bruto da Costa $(9,10)$, adoptando as directivas do autor para a mensuração da pobreza. Consideramos a linha de pobreza determinada na Europa, segundo a qual é considerado pobre um indivíduo que viva numa família cujo rendimento por adulto equivalente é inferior a 60 por cento do valor mediano por adulto equivalente calculado para toda a população (11), que está na base da atribuição da medida de apoio social Rendimento Social de Inserção (RSI).

Utilização de cuidados de saúde

A utilização dos cuidados de saúde éum fenómeno comportamental complexo.Os estudos empíricos mostram que a utilização está relacionada com a disponibilidade, a qualidade e os custos dos serviços, bem como a estrutura social, as crenças e as características pessoais dos utilizadores (12).

Gina Novick (13) efectuou uma revisão de literatura acerca das experiências das mulheres com os cuidados de saúde pré-natais, concluindo que os estudos com mulheres pobres mencionavam a referência a barreiras linguísticas, que impedem a comunicação sobretudo com emigrantes e mulheres com baixos níveis de escolaridade e a "barreiras internas", como sejam o medo de exames ou outros procedimentos clínicos. Como nos recordam Sheppard et al (14), a acessibilidade inclui mais do que a existência e disponibilidade dos serviços, também inclui a qualidade do relacionamento dos prestadores de cuidados com os pacientes.

\section{Saúde reprodutiva}

Na década de 80 a discussão sobre saúde materno-infantil e saúde integral da mulher evoluiu para a saúde reprodutiva (15), que representa uma ruptura com a ideia de reprodução como um dever para a situar como um direito. Sendo um conceito inovador porque incorpora explicitamente os homens na dimensão reprodutiva e inclui a noção de saúde sexual (16).

A definição de saúde reprodutiva adoptada pela Organização Mundial da Saúde (17), em 1988, foi ampliada e consolidada na Conferência Internacional sobre População e Desenvolvimento (CIPD), no Cairo, em 1994, na qual os governos se comprometeram, até à data limite de 2015, a assegurar o acesso de todas as pessoas aos cuidados de saúde primários e aos serviços de saúde reprodutiva. $\mathrm{O}$ acesso aos estes serviços e a aconselhamento, incluindo planeamento familiar, traduz-se em benefícios directos para a saúde de mães e filhos, permitindo àquelas uma participação na vida económica e social. O estatuto social é decisivo para a saúde reprodutiva e esta é decisiva para as mulheres, mas também para a geração seguinte. 
Esta investigação pretendeu contribuir para a evolução do conhecimento acerca das influências do contexto social nas representações das mulheres e dos profissionais de saúde acerca da fecundidade e a forma como estas influenciam as práticas e formas de utilização dos cuidados de saúde.

\section{MÉTODOS}

No presente estudo qualitativo foram realizados oito entrevistas individuais a mulheres com idades entre os 17 e 42 anos ( 3 muito pobres, 2 pobres e 3 não pobres). E dois grupos focais a mulheres entre os 19 e 49 anos - um com 5 mulheres ciganas ( 3 muito pobres e 2 pobres) e outro com 5 mulheres negras ( 2 muito pobres e 3 pobres). Com uma amostra total de 18 mulheres. Foram efectuados dois grupos focais a profissionais de saúde (um com 1 médico 7 enfermeiros e 21,8 anos em média de experiência profissional; outro com 8 enfermeiros e 14,5 anos em média de experiência profissional) num total de 15 participantes. Para a constituição da nossa amostra foi usado o princípio da saturação teórica (18). A necessidade de se utilizar ambas as técnicas de recolha de dados com as mulheres deveu-se à especificidade da população: resistência em expor as suas opiniões em grupo; dificuldades para efectuar as deslocações e em conseguir disponibilizar tempo nas suas vidas sobrecarregadas com muitas horas de trabalho e acumulação de tarefas familiares. Esta opção metodológica encerra a vantagem adicional de se conseguir várias perspectivas sobre a mesma situação, atingindo diversidade na informação recolhida (19). Todas as entrevistas e grupos focais foram gravados, seguindo-se a sua transcrição integral. Na análise dos dados utilizaram-se procedimentos de análise de conteúdo, procedendo-se a uma análise categorial temática (20).

\section{RESULTADOS}

Representações das mulheres

Comportamentos de fecundidade. Para todas as mulheres em estudo o melhor e o pior dos fílhos envolve três níveis:

- O nível dos sentimentos, dicotomizado entre as idealizações (filhos permitem alcançar a "felicidade"; garantem a "força para viver" ou permanecem "pequeninos" e "saudáveis") e os medos e preocupações inerentes ao "ser mãe" ("ameaças externas" depois dos filhos nascerem e das incertezas antes de nascerem).

- O nível das funções parentais, acentuando as obrigações reflectidas em tarefas domésticas diárias como "dar banho", e realçando a responsabilidade associada. E7_C2: “(...) é aquela responsabilidade (...) fazer comer, se eu fosse sozinha muitas vezes eu 
não fazia comer para mim, porque passava. Aquelas responsabilidades tipo dar jantar a elas, ter que as lavar, preparar a roupa."

- O nível das privações, incluindo aspectos limitativos como: continuar os estudos, conseguir descansar, ter diversões e passeios apenas apropriados para crianças, não comprar "roupas" para a própria e não poder trabalhar tantas horas e/ou em todos os horários.

As mulheres muito pobres, pobres, não pobres e negras enfatizaram o que designamos por centralidade na mulher antes dos fílhos, com tempo e prioridades defínidas em função das próprias e ênfasenos objectivos individuais. E8_C2: "Depois de ter filhos nunca mais fui a uma discoteca para conviver. Quer dizer, depois de ter filhos, tive filhos com 22 anos, dali nunca mais fui a uma discoteca. Se não vou deixar os meus filhos, era divertido, eu lá a me divertir, acontece qualquer coisa. A partir dali parou-se adiscoteca. Não é que eu não goste de festas, mas festas é só em convívios com os familiares. Depois dali, parou-se tudo"

Para as mulheres ciganas, pelo contrário, o desejo de ser mãe é colocado como o aspecto essencial da vida, mesmo antes dos fílhos. GF_M1: “(...) eu nunca pensei nisso ... a minha vida sem filhos não era nada".

Quanto à vida depois dos filhos, na opinião geral existe um desvio da centralidade da mulher para os filhos, que se traduz em expressões como "esquecemos de nós"; "não ter tempo para nós"; "temos que dedicar mais aos filhos, já não me dedico tanto a mim". Mas também há mulheres que se conseguem preparar para as mudanças que os filhos implicam, denotando uma visão pragmática das obrigações relativas aos filhos e uma relação com a existência de planeamento dos mesmos.

Planeamento familiar. As mulheres não receberam informação sobre as formas de controlo de fecundidade / contracepção por parte das suas mães, a maioria obteve as primeiras informações através de amigos, média ou de vizinhos. E6_C2: “(...) amigas. (...) pessoas externas à família, porque em casa sempre foi assunto tabu.”

Para muitas das mulheres o planeamento aconteceu apenas no seguimento das primeiras gravidezez e não de forma "preventiva", no sentido de que os filhos acontecessem de forma planeada.Realçamos as especificidades das mulheres ciganas: não utilizaram ou utilizam consultas de planeamento familiar, havendo "conselhos" por parte das vizinhas. A contracepção é um assunto que fica a seu cargo e "sem eles saberem" [os companheiros]. GF_M1: "Eu tomava assim, sempre, era bom aquilo, era seguro, era uma injecção, que eu tomava sem ele saber, todos os meses, ele nunca foi senhor de saber e depois quando tinha o meu filho maior deixava de tomar para engravidar". 
Percepção sobre a necessidade de cuidados de saúde matema. Na opinião das participantes ciganas e negras a gravidez é vista como algo "normal", logo os cuidados a ter estão condicionados por essa percepção. Mas uma das mulheres ciganas devido a problemas de saúde demonstra um conhecimento, uma preocupação e práticas de utilização bastante diferentes das restantes. GF_M1: “... a mulher numa gravidez não está doente.(...) eu penso que também a gente deve ter alguns cuidados ... não estamos doentes, mas se eles mandarem a gente deve fazer."

Quanto aos cuidados de saúde pré-concepcionais, não se registam diferenças, ou seja, de uma forma geral não tiveram esses cuidados nos primeiros fílhos e apenas as mulheres que são seguidas em termos de planeamento familiar revelaram ter preparado as últimas gravidezes.

Relativamente à vigilância pré-natal todas as mulheres conhecem e destacam a importância da sua utilização. Os cuidados que deverão ser seguidos durante as gravidezes são: alimentação, não mexer em detergentes, não comer marisco, fazer as análises e "tudo o que os médicos mandam".

No que concerne aos cuidados de saúde após o parto a situação é diferente, mesmo detendo a informação e o conhecimento da sua existência, nem todas fizeram as consultas. Algumas das razões apontadas foram: a vergonha e a revisão do parto não ser necessária quando se trata de "parto normal".

Barreiras à utilização cuidados de saúde materna. Para a grande maioria das mulheres não houve dificuldades na compra de medicamentos e/ou nos exames durante a gravidez. Tal facto está relacionado com a não existência de despesas associadas com a vigilância pré-natal no Serviço Nacional de Saúde.

Apenas houve referência por uma mulher não pobre a dificuldades associadas a uma gravidez de risco, com implicações para a compra de medicamentos e consultas. Mas esta sobrecarga económica representada pela gravidez de risco relaciona-se com os custos elevados da opção de consultas na Medicina Privada (considerando o atendimento melhor no sector privado).

Uma outra entrevistada mencionou o "medo" como factor decisivo para ter faltado à amniocentese, revelando-se ainda a falta de informação como uma barreira à realização de um exame. E8_C2: “(...hã...) faltei porquê? Porque tinha medo (...) eu disse "se falha a agulha e furar alguma coisa do meu filho que está lá na barriga" [risos da entrevistada]. Pronto, cada um pensa como quer, nãoé e não fui a esse exame." 
Relacionamento com os profissionais de saúde. Todas as mulheres se sentiram bem tratadas pelos médicos no decorrer das suas gravidezes. Houve dois aspectos que emergiram: por um lado, o desconforto de algumas mulheres por serem vistas por homens médicos; por outro, o incómodo causado por não serem transmitidas informações - quando discutiam a situação clínica e não a explicaram à mulher e ser indicada para cesariana sem explicarem a razão. E7_C2:"(...) na hora do parto não. Mas nas consultas eu tinha muita vergonha. (...) sempre fui bem tratada (...). Eu tinha era muita vergonha, chegar ali, abrir as pernas e depois o médico estar ali a ver e a tocar. Ficava muito envergonhada mesmo. Ficava nervosa, mas na hora do parto não."

Existe uma diferença nítida entre compreender e aceitar as recomendações dos profíssionais de saúde, nomeadamente da vigilância pré-natal e revisão do parto. Ou seja, todas as mulheres compreenderam as indicações terapêuticas e/ou recomendações dos profíssionais de saúde, mas muitas delas assumem que não as seguiram: "não fazemos tudo o que as médicas mandam". Os exemplos são diversos: não tomar um determinado medicamento; não fazer a consulta de revisão do parto; não fazer a aminiocentese; não seguir as indicações sobre restrições alimentares; não cumprir a recomendação médica sobre o plano alimentar da criança.

Representações dos profissionais de saúde

Comportamentos de fecundidade e planeamento familiar. Verifica-se uma tensão entre a opinião de "não diferença" entre as mulheres pobres e as "outras" relativamente ao papel dos fílhos, para assumir as diferenças devido a "vivências", "cultura" e "valores".

Segundo os profissionais de saúde o nível de habilitações da mulher acaba por ter impacto a vários níveis: por um lado, condiciona a forma como os filhos são tratados, as vivências parentais e os cuidados que são prestados. GF_S2:"(...) cada mãe é a melhor mãe que é capaz de ser... acho que não há más mães, há mães menos competentes do que outras e muitas vezes aliado à pobreza há também a iliteracia" $\mathrm{E}$, por outro, condiciona também o papel que as mulheres se auto-atribuem. GF_S1:“(...) éuma moçanormalíssima que nunca estudou, mas o papel dela também é ter filhos"

Nesta perspectiva é ainda referida, a título de exemplo, a questão da gravidez na adolescência e a forma como as jovens encaram esse papel dos filhos nas suas vidas, como meio de se afirmarem e emanciparem, percepção muito de acordo com a literatura (21). GF_S2: “(...) a gravidez na adolescência para nós profissionais de saúde é um problema, é um drama, para eles não o é porque, no fundo, nas pessoas com um nível de rendimento mais baixo é a única coisa que elas têm e que podem chamar que é delas, a gravidez é sentida como algo que eu tenho meu e só meu". 
Os profíssionais de saúde relacionam ainda os baixos níveis de literacia das mulheres às dificuldades de compreensão da informação que transmitem. GF_S1: “(...) mas para a pessoa assimilar a informação é preciso a pessoa ter uma certa literacia .... [há pessoas que começam por iniciativa própria] ... [muitas vezes vão à farmácia e compram uma pílula]... e o nível de iliteracia é bastante grande".

Uma característica apontada a estas mulheres é o "imediatismo", que se traduz na forma como fazem ou não planeamento familiar (mas também como se relacionam com os cuidados de saúde com elas e com os filhos). GF_S1:"(...) tem que ser mesmo uma insistência permanente para as conseguir sensibilizar, porque elas são muito imediatas, vêem tudo muito no imediato e ... não conseguem ... porque não foram escolarizadas".

As diferenças étnicas são novamente destacadas nas práticas de controlo de fecundidade, enfatizando-se as especificidades grupais nos cuidados de saúde.

São realçadas as dificuldades na utilização da contracepção, nomeadamente os erros e falhas associados à toma da pílula: não fazer a pausa; tomar assim que aparece a menstruação; esquecimento; tomar toda a carteira de uma só vez e tomar a pílula concomitantemente com antibiótico.

Cuidados de saúde materna. Segundo os profíssionais não há diferenças entre as mulheres pobres e as outras utentes quanto à vigilância da gravidez, embora realcem a influência dos percursos de vida nas formas de utilização dos cuidados de saúde. É referido o facto de ser feita pouca utilização de consultas préconcepcionais. GF_S1: “(...) o pré concepcional pouco, na nossa realidade (...) mas a noção que eu tenho é que estas mulheres aparecem grávidas [sim, sim] ... aí o pré-concepcional não tem significado".

Para os profissionais de saúde o que determina a diferença na vigilância prénatal não será o nível económico da mulher, mas antes "a vontade" de ter aquele fílho ou não. Sendo reconhecida a grande melhoria em todo o acompanhamento efectuado na área da saúde materna.

A realidade continua a pautar-se por pouca utilização das consultas de revisão do parto, apesar das melhorias. As razões prendem-se com uma não valorização do cuidar de si que, tendencialmente, a mulher manifesta no período pós-parto. GF_S1: "sim, a revisão do parto é má, em vez de vir na data certa vêm depois". Também em relação aos cuidados de saúde, existem especificidades relacionadas com a etnia cigana, que são uma vez mais realçadas. 
Motivações para a utilização dos cuidados de saúde. Para os profíssionais a utilização dos cuidados de saúde, durante o período da gravidez e com os filhos, acontece com pressupostos de "negociação". Isto é, o acompanhamento de saúde em troca de "um papel", o comprovativo para: o RSI; as vacinas dos filhos para entregar na escola; saber o sexo do bebé; provar que estão grávidas para receber o abono pré-natal. GF_S1: "[eles nisso não cumprem nada!] ... [só quando sentem ali pressionados... quando sentem a necessidade absoluta do papel] ... Pois, quando vão para a escola porque é obrigatório as vacinas e para entrar na escola".

Registamos a importância dos conhecimentos transmitidos no contexto familiar, partilhando experiências e práticas na utilização dos cuidados de saúde.

Podemos agrupar os factores de utilização dos cuidados de saúde relacionados com os serviços de saúde em duas áreas:

- Relacionamento profissional/utente, com os seguintes condicionalismos: dificuldades de comunicação impostas pela diferença, neste caso linguística, associada à população emigrante; necessidade de tempo para estabelecer uma relação de confiança (importância do trabalho comunitário, que é favorecedor deste tipo de relacionamento). GF_S2: "não sei se esta alteração do comportamento desta senhora passa pela necessidade que ela sentiu em vir vigiar a gravidez ou pela relação que ela estabeleceu com as pessoas... com os técnicos, porque eu acho que eles podem-se aproximar e vão-se aproximando, a relação é fundamental [ah, sim, mas isso em qualquer pessoa]".

- Organização dos serviços, que nem sempre se coaduna o "imediatismo" destas populações. Por exemplo, a dificuldade de se conseguir "convencer" as mulheres a ir fazer uma consulta e depois não existir resposta por parte dos serviços. Este ponto não foi consensual, sendo dado o contraponto dos comportamentos de utilização inadequados por parte das populações - por exemplo, não comparecer a consultas marcadas ou não avisar quando têm que faltar.

Três tipos de barreiras à utilização de cuidados de saúde. 1. Forma como as mulheres se relacionam com o próprio corpo (independentemente do nível socioeconómico) e os constrangimentos à utilização de consultas de ginecologia / obstetrícia realizadas por médicos homens, realçando os aspectos associados à "vergonha de expor o corpo" e de "ser tocada". Mas esta não foi uma barreira considerada por todos os participantes, alguns consideraram-na "ultrapassável" e passível de "ser negociada"; 2. O papel da família como incentivador, ou não, de utilização de cuidados de saúde, nomeadamente, no que diz respeito à vigilância pré-natal; 3. Afalta de informação, que é uma das características destas mulheres 
e influencia a forma como se relacionam com os cuidados de saúde, podendo mesmo constituir-se como uma barreira à utilização dos cuidados de saúde materna.

\section{DISCUSSÃO}

A ambivalência que caracteriza a situação de gravidez, ora percepcionada positivamente, como um "privilégio" e motivo de "vaidade", ora negativamente os medos, riscos e incertezas quanto a saber se o bebé está ou não a desenvolverse normalmente, já havia sido descrita por Atkinson e Farias (22).

Os nossos dados são consonantes com os de outros estudos nacionais. Silva (23) já antes descreveu, quer o facto de o interesse e a atenção que as pessoas dão ao corpo aumentarem à medida que se sobe na hierarquia social, quer a importância que os filhos assumem nas vidas das mulheres de etnia cigana. Wall et al (24) têm vindo a alertar para a necessidade de apoios familiares para cuidar das crianças, nomeadamente as famílias monoparentais e as mulheres com dificuldades económicas, quando não há ajudas informais e revelam acesso difícil a apoio público de creches, que são instituições muito requisitadas. Este facto assume no caso das mães sós contomos de grande vulnerabilidade, pelo que é preciso ter em atenção a estratificação das famílias monoparentais.

É necessário ainda lembrar que os dados relativos a Portugal mencionam uma melhoria considerável nos indicadores, mas também a persistência de números elevados de não vigilância e de vigilância tardia da gravidez $(2,25,26)$.

Os nossos resultados vão também no mesmo sentido da literatura internacional. Patrick e Bryan (27) chamaram a atenção para os stressores da gravidez e da maternidade, como a perda de emprego e redução dos horários de trabalho. Kendall et al (21) dizem-nos que o desejo para reivindicar a maioridade, desenvolver a estabilidade familiar e conjugal, e conseguir maior intimidade com o parceiro são motivações mais fortes do que os modelos idealizados de carreira e casamento. Zabin (28) descreve as situações em que as oportunidades de carreira não existem na maior parte das vezes e os pais dos bebés não estão muitas vezes presentes, enfatizando a realidade das famílias monoparentais femininas, muito presente nestes contextos de pobreza. Cockerham (29) assinala o facto de os pobres serem mais imediatos nas suas acções, tal como a nossa característica-tipo: "imediatismo", atribuída às mulheres pobres, como traço identitário presente em praticamente todas as suas acções, desde o entendimento que as mulheres pobres têm dos filhos e da fecundidade, que se cruza com as práticas de planeamento familiar, de utilização de 
contracepção e de planeamento dos filhos, mas também de utilização dos cuidados de saúde.

Whitehead e Szelag (30) demonstraram que em algumas culturas a gravidez é vista como uma etapa normal da vida, não uma patologia que necessite de intervenção. Estes autores acentuam que as atitudes e decisões num mesmo grupo cultural / étnico nunca são homogéneas. E os seus estudos apontaram ainda o desconforto durante o encontro biomédico nos cuidados pré-natais resultando de uma incapacidade da mulher para lidar com certas características do profissional de saúde, nomeadamente idade, género e linguagem. Também Campbell et al (31) nos dizem que a necessidade de cuidados de saúde prénatais varia de acordo com os contextos sociais e culturais, traduzindo-se na forma como entendem a gravidez e a necessidade de consultas pré-natais.

Chamamos a atenção para resultados como a obtenção das primeiras informações sobre os métodos contraceptivos através de amigos, dos média e/ ou de vizinhos; a percepção generalizada de que o sexo foi um assunto tabu na educação das mulheres e a preparação inadequada para o sexo e contracepção na primeira gravidez, acontecendo esta como resultado de impreparação e não de planeamento $(21,32-34)$. Estes mesmos autores mencionam que os métodos hormonais são entendidos como produzindo um desgaste físico nos corpos das mulheres e a toma continuada e sistemática é muitas vezes interrompida devido a faltas a consultas, a eficácia dos métodos é questionada, o conhecimento e uso dos mesmos é inadequado e o relato de falhas nos preservativos é elevado $(21,32-$ 34). Segundo alguns destes autores (21) o planeamento efectivo e os comportamentos necessários para evitar a gravidez ocorrem apenas quando existem motivações fortes e ambientes que apoiem a manutenção do estado de não-gravidez.

Este nosso trabalho surge enquadrado em estudos que realçam a importância de conhecer as características e as percepções sobre as necessidades de cuidados de saúde materna por parte de grupos específicos, como as mulheres ciganas, no sentido de conseguir elaborar medidas específicas e efectivas de sensibilização para a utilização dos cuidados de saúde adequados às necessidades das populações (35). Em jeito de conclusão, diríamos que por forma a alcançar a equidade na área da saúde será necessário haver uma intervenção intersectorial

Agradecimentos: Esta investigação teve o apoio da Fundação para a Ciência e TecnologiaPOCI 2010, Fundo Social Europeu (SFRH/BD/2004). Os autores agradecem ainda o apoio da Santa Casa da Misericórdia de Lisboa (SCML), Centro de Malária e Outras Doenças 
Tropicais (CMDT) e AGO (Associação para a Cooperação e Desenvolvimento Garcia D’Orta).

\section{REFERÊNCIAS}

1. Santana P. Poverty, Social Exclusion and Health in Portugal. Social Science and Medicine 2002; 55: 33-45.

2. Portugal, Direcção Geral da Saúde, Saúde, Sexo e Género-factos, representações edesafios. Vasco Prazeres (coord.). DGS, Lisboa; 2008.

3. Furnham A. Lay Theories-everyday understanding of problems in the social sciences.Oxford Pergamon Press; 1988.

4. Jodelet D. Représentations Sociales: un domaine en expansion. In: Jodelet D. LesReprésentations Sociales. P.U.F. Paris; 1989.

5. Braveman P, Gruskin S. Defining equity in health. Journal of Epidemiology and Community Health. 2003; 57: 254-258.

6. Mackenbach J, Martijntje JB. Tackling socioeconomic inequalities in health: analysis of European experiences. The Lancet. October 25 2003; 362:1409-1414.

7. Braveman P. Health disparities and health equity: concepts and measurements. Annual Review of Public Health. 2006; 27: 167-194.

8. Whitehead M, Dahlgren G. European strategies for tackling social inequities in health: leveling up part 2. Studies on social and economic determinants of population health, $\mathrm{N}^{\circ} 3 . \mathrm{WHO}$ Regional Office for Europe, Copenhagen. 2007 [Internet]. Disponible en: http:// www.euro.who.int/document/e89384.pdf Acedido em Agosto de 2009.

9. Bruto da Costa A. Exclusões Sociais. Colecção Fundação Mário Soares, Gradiva, Lisboa;1998.

10. Bruto Da Costa A. (Coord.). Um olhar sobre a Pobreza: vulnerabilidade e exclusão social noPortugal contemporâneo. Gradiva, Lisboa; 2008.

11. Rodrigues CF. Distribuição do Rendimento, Desigualdade e Pobreza. Portugal nos anos $90 . \mathrm{N}^{\circ}$ 5 da Colecção Económicas. Ed. Almedina, Coimbra; 2008.

12. Chakraborty N, Islam MA, Chowdhury RI, Bari W, Akhter HH. Determinants of the use of maternal health services in rural Bangladesh. Health Promotion International. 2003; 18(4): 327337.

13. Novick G. Women experience of prenatal care: an integrative review. Journal of MidwiferyWomen's Health. 2009; 54(3): 226-237.

14. Sheppard VB, Zambrana R, O'malley AS. Proving health care to low-income women: amatter of trust. Family Practice. 2004; 21 (4):484-491.

15. Correa S, Jannuzzi PM, Alves JED. Direitos e Saúde Sexual e reprodutiva: marco teóricoconceitual e sistema de indicadores, 2003; p.1-16 [Internet]. Disponible en: www.abep.org.br/fotos/Dir_Sau_Rep.pdf Acedido em Agosto de 2009.

16. CIDM (Comissão para a Igualdade e para os Direitos das Mulheres). Plataforma de Acção dePequim, 1995 , Iniciativas e Acções Futuras: Igualdade de Género, Desenvolvimento e Paz para oSéculo XX, Agenda Global n 5, Presidência do Conselho de Ministros, Lisboa; 2001.

17. WHO. Progress in Reproductive Health Research.n $n^{\circ}$ 42, part 1, p. 1-4, [Intemet]. Disponible: www.who.int/reproductive-health/hrp/progress/42/news42_1.en.html Acedido em Agosto de 2009.

18. Guerra IC. Pesquisa qualitativa e análise de conteúdos: sentidos e formas de uso. Estoril. Princípia Editora; 2006.

19. Igea D, Agustin J, Beltrán A, Martin A. Métodos y técnicos cualitativos de investigación en ciencias sociales. Editorial Síntesis, Madrid; 1995.

20. Bardin L. Análise de Conteúdo. Lisboa: Edições 70; 2008. 
21. Kendall C, Afable-Munsuz A, Speizer I, Avery A, Schmidt N, Santelli J. Understanding pregnancyin a population of inner-city women in New Orleans-results of a qualitative research. Social Science and Medicine. 2005; 60: 297-311.

22. Atkinson S, Farias MF. Perceptions of risck during pregnancy amongst urban women in Northeast Brasil. Social Science and Medicine. 1995; 41 (11): 1577-1586.

23. Silva LF. Saúde/Doença é Questão Cultural, Atitudes e comportamentos de saúdematerna das mulheres ciganas em Portugal. Centro de Estudos das Migrações e Relações Interculturais, Universidade Aberta. Lisboa: ACIME; 2005.

24. Wall K, São José J, Correia SV. Mães sós e cuidados às crianças. Análise Social 2002; 163:631-663.

25. Alves O, Mendonça D, Maciel-Barbosa A. Saúde da mulher e da criança: necessidades, comportamentos e atitudes. Revista Portuguesa de Saúde Pública. 2005; 23(1): 69-80.

26. Delvaux T, Buekens P, Godin I, Boutsen M. Barriers to prenatal care in Europe. American Journal of Preventive Medicine. 2001; 21(1):52-59.

27. Patrick TE, Bryan Y. Research strategies for optimizing pregnancy outcomes inminority populations. American Journal of Obstetrics and Gynecology. 2005; 192: S64-70.

28. Zabin LS. Ambivalent feelings about parenthood may lead to inconsistent contraceptive use of pregnancy. Family Planning Perspectives. 1999; 31 (5): 250-251.

29. Cockerham WC. Medical Sociology. In: Smelser NJ (Ed,). Handbook of Sociology. London: Sage Publications; 1988. p. 575-599.

30. Whiteford LM, Szelag BJ. Access and utility as reflections of cultural constructions of pregnancy.Primary care update for Ob/Gyns. May 1 2000;7(3): 98-104.

31. Campbell JD, Mitchell P, Stanford JB, Ewigman BG Validating a model developed to predictprenatal care utilization. The Journal of Family Practice. 1995; 41(5): 457-464.

32. Crosby R, Sanders S, Yarber WL, Graham CA. Condom-Use Errors and Problems, ANeglected Aspect of Studies Assessing Condom Effectiveness. American Journal of Preventive Medicine. 2003; 24(4): 367-370.

33. Macaluso M, Artz L, Kelaghan J, Austin H, Fleenor M, Hook EW. Prospective study ofbarrier contraception for the prevention of sexually transmitted diseases: study design and general characteristics of the study group. Sexually transmitted diseases. March 26 1999; (3): 127-36.

34. Spruyt A, Steine MJ, Joanis, C, Glovet LH, Piedrahita C, Alvarado G, Rainos R, Maglaya C, Cordero M. Identifying Condom Users at Risk for Breakage and Slippage: Findings from Three International Sites. American Journal of Public Health. 1998; 88: 239-244.

35. Melnikow J, Alemagno SA, Rottman C, Zyzanski SJ. Characteristics of Inner-City Women Giving Birth with Little or No Prenatal Care: A case-control study The Journal of Family Practice. 1991; 32(3): 283-288. 